\title{
Rol de las características clínicas e histológicas como factores pronósticos para la sobrevida en pacientes con carcinoma de células escamosas de la cavidad oral*
}

\author{
Drs. OSCAR ARRIAGADA C. ${ }^{1}$, BERNARDO VENEGAS R. ${ }^{2}$, MARIO CANTÍN L. ${ }^{1}$, \\ DANIELA ZAVANDO M. ${ }^{3}$, CARLOS MANTEROLA D. ${ }^{4}$, IVÁN SUAZO G. ${ }^{1}$ \\ 1 Departamento de Ciencias Básicas Biomédicas, Universidad de Talca, Chile. \\ 2 Departamento de Estomatología, Universidad de Talca, Chile. \\ 3 Universidad Autónoma de Chile, sede Talca, Chile. \\ 4 Departamento de Cirugía, Universidad de La Frontera, Temuco, Chile.
}

\begin{abstract}
Oral squamous cell carcinoma. retrospective analysis of $\mathbf{3 6}$ cases

Background: Survival of patients with oral squamous cell carcinoma is low and depends mostly on TNM staging of the tumor. Aim: To perform a retrospective analysis of a series of patients with oral squamous cell carcinoma. Material and Methods: Retrospective review of patients with the diagnosis, seen between 1995 and 2006 in a regional hospital. Host inflammatory response and pattern of tumor invasion were assessed using the staging proposed by Bryne et al. Results: The medical records of 36 patients aged 39 to 89 years, were reviewed. During the study period, 15 patients died. Better survival was associated to a low pattern of tumor invasion and a high inflammatory response and the topographic location of the tumor. Conclusions: Inflammatory response, tumor invasion and location are associated with survival in oral squamous cell carcinoma.
\end{abstract}

Key words: Oral cancer, squamous cell, survival.

\section{Resumen}

Introducción: El carcinoma de células escamosas de la cavidad oral (CCECO) es una patología cuyo comportamiento es producto de interrelaciones con el huésped, esto es, por el patrón de invasión (PI) histopatológica y la respuesta inflamatoria (RI). El objetivo de este estudio es analizar las características clínicas e histopatológicas como factores pronóstico, en términos de supervivencia (SV) en pacientes con CCECO. Material y Método: Serie de casos. Se incluyeron pacientes diagnosticados en el Hospital Regional de Talca y Hospital Base de Curicó entre los años 1995 y 2006. Se revisaron las fichas clínicas y biopsias de 36 pacientes con CCECO. Se determinó el Frente Invasivo Tumoral (FIT), evaluándose los parámetros propuestos por el sistema de graduación de Bryne (PI y RI) y factores de importancia clínica como localización topográfica de la lesión, edad y género, relacionándolos con SV mediante curvas de Kaplan-Meier y Log Rank test. Posteriormente, se aplicó una regresión de Cox para obtener un análisis multivariado y cálculo de RR. Del total de

*Recibido el 22 de Marzo de 2010 y aceptado para publicación el 22 de Mayo de 2010.

Correspondencia: Dr. Iván Suazo G.

Avenida Lircay s/n oficina 104, Talca, Chile.

E-mail: isuazo@utalca.cl 
casos del estudio, 15 pacientes fallecieron por CCECO. Resultados: La mayor SV se asoció a un bajo escore de PI y una alta RI respectivamente (RR 1,5). La localización topográfica de la lesión se relacionó significativamente con la SV, no así el grupo de edad. Conclusiones: Nuestros resultados sugieren que la ubicación de la lesión es un factor importante en el pronóstico de la enfermedad y que una respuesta inmune/inflamatoria adecuada, expresada en un bajo escore de RI, mejora el pronóstico de SV en pacientes con CCECO.

Palabras clave: Carcinoma de células escamosas, cavidad oral, cáncer oral, pronóstico, supervivencia.

\section{Introducción}

El carcinoma de células escamosas de cavidad oral (CCECO) es una neoplasia proveniente de los tejidos epiteliales. Se inicia como una displasia epitelial, la cual prolifera adquiriendo características malignas, destruyendo el tejido conjuntivo subyacente. Los índices de supervivencia (SV) de esta neoplasia son bajos (50\%), especialmente si se compara con otras neoplasias ${ }^{1}$.

$\mathrm{Su}$ evaluación pronóstica y terapéutica se ha basado principalmente en la clasificación clínica $\mathrm{TNM}^{2}$, la que ha mostrado, incluso con la medicina moderna, una tasa de mortalidad general alta. Según la literatura, características clínicas como la edad del paciente y el sitio anatómico de la lesión también deben ser consideradas ${ }^{3,4}$. Según la edad del paciente, factores de riesgo como el tabaco y el alcohol, afectarían principalmente a adultos mayores debido a una prolongada exposición. En jóvenes, se demuestra una disminución o ausencia de exposición a estos factores, planteando cuestionamientos en la etiología de la enfermedad ${ }^{5}$, sugiriendo mejores índices de SV en ellos. Además, su presentación varía ampliamente en diferentes poblaciones, localizándose en diversos sitios de la cavidad oral, lo que ha mostrado una gran variabilidad en su pronóstico, repercutiendo de manera importante en la SV de los pacientes $^{2,6,7}$.

Actualmente, la consideración de la biología tumoral, específicamente sus características histológicas, ha sido utilizada para realizar un diagnóstico precoz del CCECO, teniendo un papel fundamental en su pronóstico y $\mathrm{SV}^{8}$. Las características histológicas del CCECO pueden diferir ampliamente de un área a otra dentro del mismo tumor, por lo que se cree que la información pronóstica más útil puede ser deducida del frente de invasión tumoral (FIT), zona donde residen las células tumorales más agresivas $^{9}$, considerándose la región más representativa de la neoplasia ${ }^{10}$.

Con el propósito de realizar una estimación más precisa acerca del comportamiento biológico de esta neoplasia, Bryne et $\mathrm{al}^{11}$, publicaron el sistema de graduación del FIT, en la cual, un pequeño número de variables histológicas son aplicadas a la zona de interfase entre el tumor y el huésped, demostrando ser útil en el pronóstico de la neoplasia ${ }^{12,13}$. El sistema de graduación del FIT evalúa cuatro parámetros morfológicos, otorgándole a cada uno un escore de 1 a 4, determinando el grado de malignidad para cada tumor (un alto escore sugiere un tumor altamente indiferenciado) ${ }^{11}$. Los parámetros contemplan grado de queratinización, polimorfismo nuclear, patrón de invasión y respuesta inflamatoria, donde el patrón de invasión muestra la forma en que la neoplasia se infiltra y desarrolla hacia los tejidos adyacentes, y la respuesta inflamatoria refleja la reacción inmunológica del individuo contra la neoplasia.

La relación inmunológica funcional entre respuesta inflamatoria y desarrollo del cáncer no es nueva. En 1863, Virchow, planteó la hipótesis que el origen del cáncer se encontraba en zonas de reacción inflamatoria crónica, donde la conjunción de factores como irritantes, daño tisular e inflamación desencadenaban una respuesta proliferativa celular ${ }^{14}$. Por tanto, el comportamiento neoplásico será producto de interrelaciones entre el huésped y el tumor ${ }^{15}$, donde el crecimiento neoplásico está invariablemente relacionado con la presencia de células inflamatorias en el sitio primario del tumor ${ }^{16}$. Diversos estudios han demostrado que células inflamatorias contribuirían al proceso de invasión y metástasis tumoral, lo que se relacionaría directamente en como infiltra la neoplasia según el patrón de invasión. Sin embargo, el efecto real de la respuesta inflamatoria local ante la invasión neoplásica aún es controversial ${ }^{17}$.

El objetivo de este estudio es analizar las características clínicas de edad y localización tumoral, e histopatológicas de patrón de invasión neoplásico (PI) y respuesta inflamatoria del huésped (RI), como factores pronósticos en términos de $\mathrm{SV}$ en pacientes con CCECO diagnosticados en el Hospital Regional de Talca y Base de Curicó entre los años 1995 y 2006.

\section{Material y Método}

Estudio de serie de casos retrospectiva que incluyó a los pacientes diagnosticados con carcinoma de células escamosas de la cavidad oral (CCECO) en los hospitales Regional de Talca y Base de Curicó en el período enero de 1995 y diciembre de 2006. 


\section{Protocolo de estudio}

En relación al análisis histopatológico, cada pieza histológica fue teñida con la técnica hematoxilinaeosina (H\&E) para, posteriormente ser observadas bajo un microscopio Carl Zeiss 0.25 (Germany). El examen microscópico fue realizado con aumentos de 10X y 40X por un observador con conocimientos de análisis histopatológico, identificando el frente tumoral en cada una de ellas. Para la valoración de la respuesta inflamatoria local del huésped (RI) y el patrón de invasión (PI) en cada caso, se recurrió al sistema de graduación propuesto por Bryne et a ${ }^{11}$, descrito en la Tabla 1.

La información de las variables demográficas y clínicas fue obtenida desde las fichas clínicas existentes en cada hospital. Se confeccionó una hoja de recogida de datos en las que se registraron las características biodemográficas y clínicas de los pacientes, el diagnóstico confirmatorio histopatológico de CCECO, las características de las muestras histopatológicas de tamaño y estado de conservación adecuado para el análisis posterior, los datos respecto de muerte y su etiología. Una fuente adicional de registro fue la base de datos del Registro Civil e Identificación, estableciéndose de manera fidedigna que el $100 \%$ de las muertes en nuestra muestra se debió a CCECO.

\section{Variable de interés}

La variable resultado principal fue la SV de los pacientes, la que se trabajó de forma dicotómica definiéndose: Grupo 1: Adultos menores de 65 años y Grupo 2: Adultos con edad mayor o igual a 65 años la clasificación. La variable de respuesta en este estudio se construyó con base en el tiempo transcurrido entre el momento del diagnóstico de CCECO realizado por histopatología y la ocurrencia de la muerte, mientras que la variable de censura se definió a partir de los pacientes que sobrevivieron durante el período de estudio.
En relación a la ubicación del tumor primario, ésta se describió de acuerdo a la región topográfica donde se encontró la mayor parte de la lesión.

\section{Plan de análisis}

Se realizó un análisis exploratorio de los datos; posteriormente, se utilizó estadística descriptiva con cálculo de porcentajes, medidas de tendencia central y dispersión (promedios y desviación estándar; mediana y valores extremos). Posteriormente se confeccionaron curvas de SV de Kaplan Meier y se aplicó Log Rank test para evaluar las diferencias entre las curvas de SV. Finalmente, se aplicó una regresión de Cox para obtener un análisis multivariado y cálculo de riesgo relativo (RR) con sus respectivos intervalos de confianza de $95 \%$.

\section{Aspectos éticos}

Se garantizó el principio ético de confidencialidad a los pacientes codificándose sus nombres. Este estudio contó con la aprobación del comité de ética de la Universidad de Talca.

\section{Resultados}

En el período de estudio fueron estudiados según este esquema 36 pacientes, sin registrarse pérdidas de seguimiento. La edad promedio fue de 64,4 \pm 10,9 años (39 a 89 años); y el $86,2 \%$ de los pacientes (31 casos), era de género masculino.

De la serie de casos analizados, la SV global de los pacientes fue de $58,4 \%$, mientras que la mortalidad específica por CCECO fue de 41,6\%. La curva de SV de Kaplan Meier (Figura 1) muestra el comportamiento de los grupos definidos por la variable sitio anatómico donde se localizaba la lesión, al análisis inferencial de Log Rank se demuestra significación estadística para esta variable, el detalle se presenta en Tabla 2.

Tabla 1. Clasificación de los parámetros patrón de invasión y respuesta inflamatoria según el sistema de graduación propuesto por Bryne

\begin{tabular}{lllll}
\hline $\begin{array}{l}\text { Característica } \\
\text { morfológica }\end{array}$ & \multicolumn{1}{c}{ Score } \\
\cline { 2 - 4 } & \multicolumn{2}{c}{$\mathbf{2}$} & $\mathbf{3}$ & \multicolumn{1}{c}{$\mathbf{4}$} \\
\hline Patrón de invasión & $\begin{array}{l}\text { Bordes bien delimi- } \\
\text { tados }\end{array}$ & $\begin{array}{l}\text { Infiltrativo. Cordones, } \\
\text { bandas y/o acintados }\end{array}$ & $\begin{array}{l}\text { Pequeños grupos o } \\
\text { cordones de células } \\
\text { infiltrantes }(\mathrm{n}>15)\end{array}$ & $\begin{array}{l}\text { Disociación celular } \\
\text { marcada y desorga- } \\
\text { nizada en pequeños } \\
\text { grupos y/o células } \\
\text { solas }\end{array}$ \\
$\begin{array}{l}\text { Respuesta } \\
\text { inflamatoria }\end{array}$ & Marcada & Moderada & Leve & Ausente \\
\hline
\end{tabular}




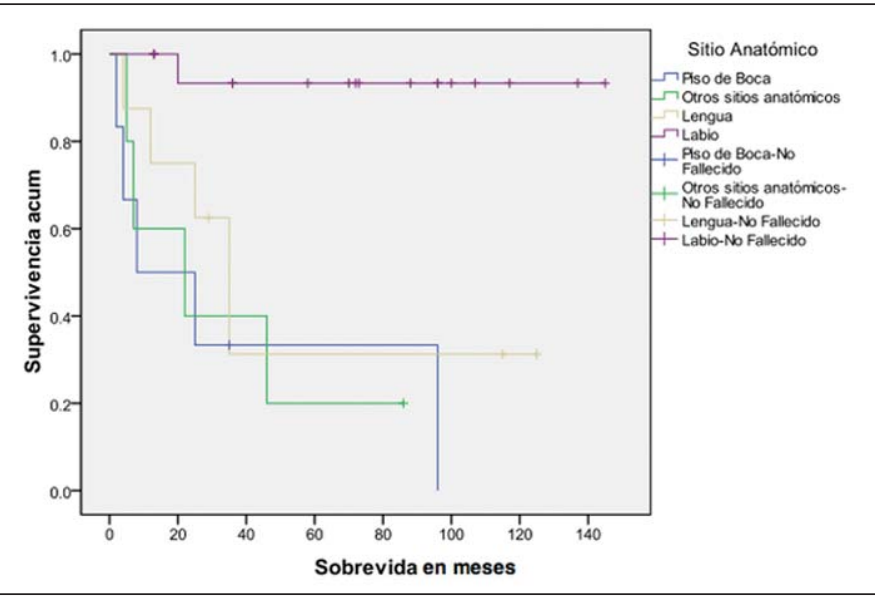

Figura 1. Curva de supervivencia en relación al sitio anatómico.

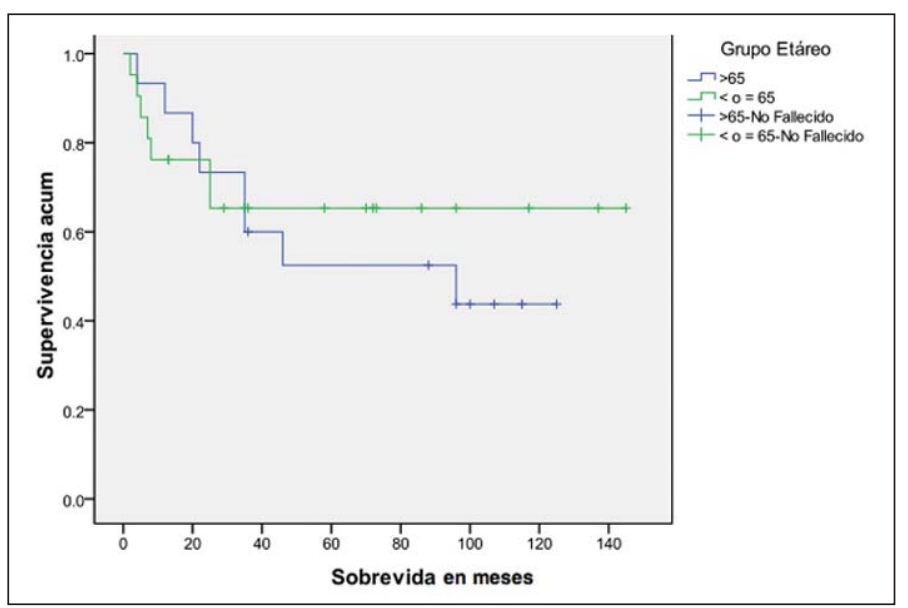

Figura 2. Curva de supervivencia en relación al grupo de edad.

Tabla 2. Distribución de fallecidos por CCECO de acuerdo al sitio anatómico de la lesión y estadística analítica de su relación con la SV

\begin{tabular}{lcccc}
\hline Sitio anatómico & n total & Fallecidos & \multicolumn{2}{c}{$\begin{array}{c}\text { Log Rank } \\
\text { (Mantel-Cox)* }\end{array}$} \\
\hline Piso de boca & 6 & 5 & & \\
$\begin{array}{l}\text { Otros sitios } \\
\text { anatómicos }\end{array}$ & 5 & 4 & $\chi^{2}$ & 18,145 \\
Lengua & 8 & 5 & $\mathrm{gl}$ & 3 \\
Labio inferior & 17 & 1 & Sig. & 0,000 \\
Global & 36 & 15 & & \\
\hline
\end{tabular}

*Prueba de igualdad de distribuciones de supervivencia para diferentes sitios anatómicos.
Al analizar el rol de la edad al momento del diagnóstico, se observa que los grupos contrastados no presentan diferencias estadísticamente significativas respecto de la $\mathrm{SV}$, el detalle de estos datos se encuentra en la Tabla 3 y se visualizan en la Figura 2.

Los resultados de los parámetros propuestos por Bryne, esto es, respuesta inflamatoria y patrón de invasión se observan en la Tabla 4. La SV de los pacientes según agrupamiento de PI en PI 1 y 2 y PI 3 y 4 son presentados en la Figura 3. Los resultados de SV respecto de la RI, se observan en la Figura 4.

En relación al análisis, como factores pronósticos por el método de Cox, los resultados se observan en la Tabla 5 .

\section{Discusión}

El CCECO actualmente se reconoce como una patología de importancia, con una incidencia anual estimada de 170.000 nuevos casos en todo el mun$\mathrm{do}^{5}$. Su diagnóstico temprano, tiene un rol fundamental en su pronóstico y SV de los pacientes, mientras que los diagnósticos en etapas avanzadas tienen altos niveles de mortalidad. En la serie de casos analizados en este estudio, la SV global fue del 58,4\%, en concordancia con lo reportado en otros países desarrollados, con porcentajes entre el $30 \%$ y $80 \% \%^{4}$, mientras que la mortalidad específica fue de 41,6\%, similar a la tasa mundial, cercana al $50 \% 0^{2}$, demostrando que estos valores de mortalidad son elevados para esta patología.

Con el fin de entregar una información pronóstica más precisa en el curso del CCECO, se han desarrollado nuevos sistemas de graduación histológica para definir el grado de malignidad de una neoplasia, siendo el sistema de graduación histológica propuesto por Bryne el que ha demostrado en conjunto tener un alto valor pronósti$\mathrm{co}^{6,10,11,18-21}$. De los parámetros analizados en este sistema de graduación, los valores patrón de invasión y respuesta inmune local han demostrado entregar información pronóstica para diversos 
tipos de cánceres ${ }^{6,11,19,22,23}$. Junto con ellos, indicadores clínicos, como el sitio de ubicación de la lesión primaria y la edad del paciente son importantes para evaluar el pronóstico de sobrevida de los enfermos de CCECO.

En nuestro estudio analizamos el valor pronóstico de indicadores clínicos como el sitio de lesión y la edad del paciente, así como también indicadores histológicos como la respuesta inflamatoria y el patrón de invasión.

De los indicadores clínicos analizados, sólo la localización de la lesión resultó relevante como factor pronóstico, las lesiones ubicadas en el labio inferior presentaron una mayor SV que las ubicadas en otras regiones, esto sugiere que condiciones como las características del epitelio, la vascularización y el drenaje subyacente, pueden estar implicadas en un mejor pronóstico de $\mathrm{SV}$, esto es semejante a los reportado en estudios anteriores ${ }^{24-26}$.

Al evaluar el valor pronóstico para la SV de los indicadores histológicos, observamos que sólo la respuesta inflamatoria se relacionó con una mayor sobrevida (RR 1,519). Los datos obtenidos para RI fueron semejantes a lo reportado para otros tipos de cáncer primarios epiteliales ${ }^{27}$. Nuestros resultados se contraponen a lo reportado por Muñoz-Guerra et $\mathrm{al}^{28}$ y Crissman et $\mathrm{al}^{29}$, para quienes el único dato histológico de importancia en el pronóstico de SV es el patrón de invasión.

Según nuestros resultados, los pacientes que muestran un PI escore 1 y 2 tienen mayor posibilidad de sobrevivir (valor máximo 145 meses) que el PI escore 3 y 4 (valor máximo 115 meses). Bryne et al ${ }^{18}$, reportó $\mathrm{SV}$ a los 5 años del 70\% y 30\% ante el patrón de invasión escore 3 y 4 respectivamente. Kademani et $\mathrm{al}^{30}$, encontraron que las diferencias de SV entre los escore $1 \mathrm{y}$ 4 son claramente distinguibles, donde a los 5 años se encontró una SV de $80 \%, 54 \%, 41 \%$ y $29 \%$ para los scores $1,2,3$ y 4 respectivamente. Por tanto, neoplasias que invaden como cordones sólidos presentan un mejor pronóstico en términos de sobrevida que los casos con invasión en cordones pequeños o difusos, correlacionándose directa-

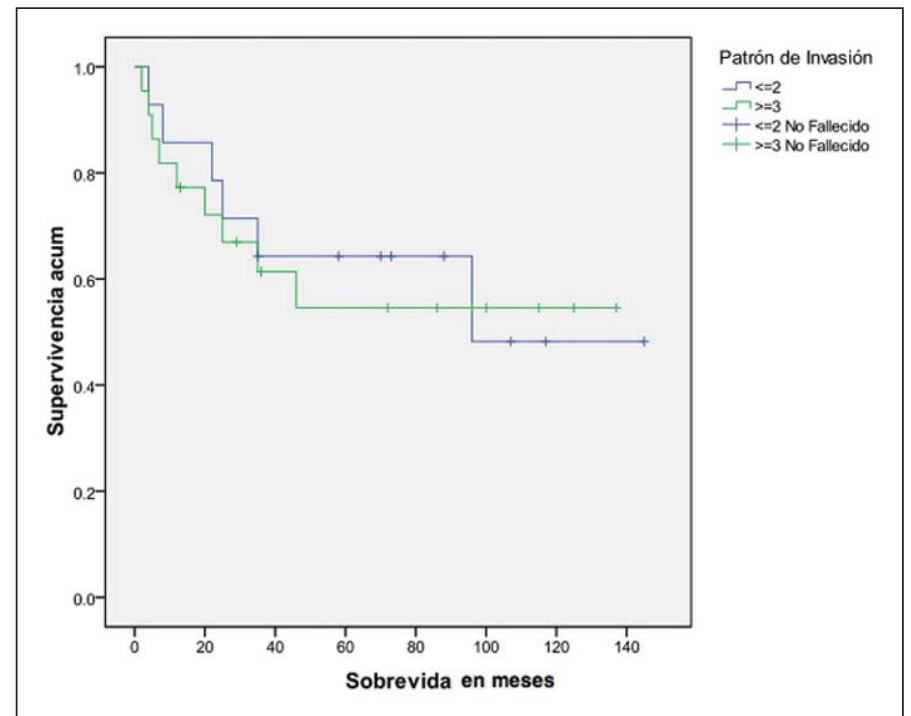

Figura 3. Curva de supervivencia en relación al patrón de invasión, clasificadas de acuerdo al escore en $\leq 2 \mathrm{y} \geq 3$.

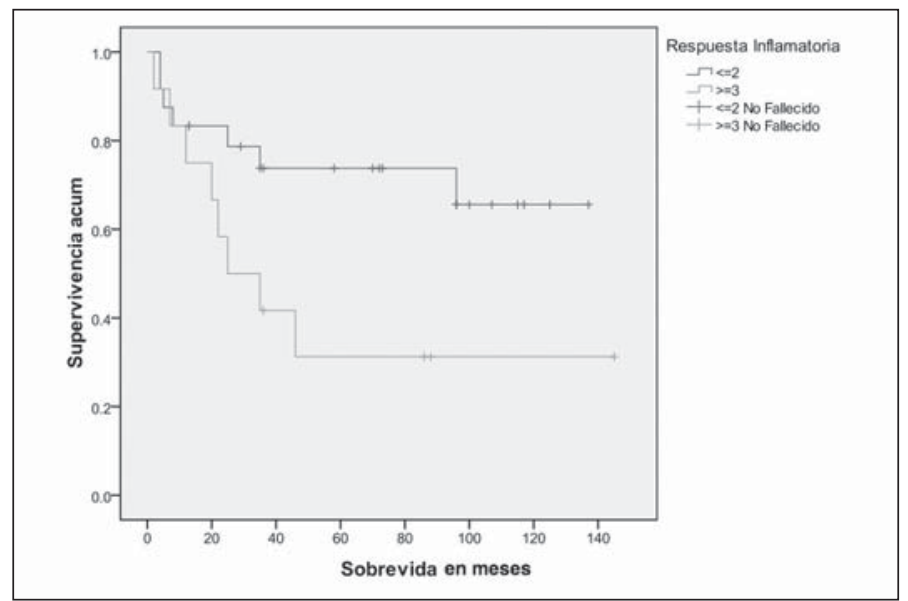

Figura 4. Curva de supervivencia en relación a la respuesta inflamatoria, clasificadas de acuerdo al escore en $\leq 2 \mathrm{y} \geq 3$.

Tabla 3. Distribución de fallecidos por CCECO de acuerdo a la edad y estadística analítica de su relación con la SV

\begin{tabular}{lcccc}
\hline Edad & n total & Fallecidos & \multicolumn{2}{c}{$\begin{array}{c}\text { Log Rank } \\
\text { (Mantel-Cox)* }\end{array}$} \\
\hline$>65$ años & 15 & 8 & Chi $^{2}$ & 0,304 \\
$\leq 65$ años & 21 & 7 & $\mathrm{gl}$ & 1 \\
Global & 36 & 15 & Sig. & 0,581
\end{tabular}

* Prueba de igualdad de distribuciones de supervivencia de acuerdo a la edad. 
Tabla 4. Detalle de los escores de respuesta inflamatoria y patrón de invasión, observados desde el frente de invasión en muestras de sujetos vivos y fallecidos

\begin{tabular}{lcccccccc}
\hline & \multicolumn{3}{c}{ Respuesta inflamatoria } & & \multicolumn{5}{c}{ Patrón de invasión } \\
& Score 1 & Score 2 & Score 3 & Score 4 & Score 1 & Score 2 & Score 3 & Score 4 \\
\hline Vivos & 8 & 9 & 3 & 1 & 1 & 7 & 10 & 3 \\
Fallecidos & 4 & 3 & 8 & 0 & 2 & 4 & 8 & 1 \\
Total & 12 & 12 & 11 & 1 & 3 & 11 & 18 & 4 \\
\hline
\end{tabular}

Tabla 5. Resultado del estudio multivariante para SV

\begin{tabular}{lccc}
\hline & $\begin{array}{c}\text { Coef. } \\
\text { Covar }\end{array}$ & Sig. & RR \\
\hline Sitio anatómico & $-0,398$ & 0,063 & 0,672 \\
Edad & 0,189 & 0,736 & 1,208 \\
Patrón de invasión & $-0,146$ & 0,654 & 0,864 \\
$\begin{array}{l}\text { Respuesta } \\
\text { inflamatoria }\end{array}$ & 0,418 & 0,166 & 1,519 \\
\hline
\end{tabular}

mente con un mayor riesgo de metástasis ${ }^{31}$. Esto se debe probablemente a que las neoplasias al presentar una mayor difusión del patrón de invasión (mayor escore), se infiltran el estroma más rápidamente e invaden los vasos linfáticos con mayor frecuencia, representando una cohorte más agresiva ${ }^{32}$.

Al analizar la respuesta inflamatoria local, se esperaba encontrar una menor SV en pacientes con un bajo escore en respuesta inflamatoria (mayor respuesta), según lo reportado previamente ${ }^{14,16,33}$, ya que el infiltrado inflamatorio representa la respuesta del estroma en presencia de neoplasias malignas y son considerados como una expresión de las interacciones entre el sistema inmune del huésped y el tumor ${ }^{34}$, donde el número y tipos de células que componen la respuesta inflamatoria corresponden de manera predominante a macrófagos, linfocitos, células natural killer, eosinófilos y mastocitos, quienes en conjunto están relacionados con la producción local de quimiocinas y otros factores quimiotácticos que aumentan las respuestas en el tumor ${ }^{35}$. En el cáncer de mama $^{36}$, de cuello del útero ${ }^{37}$, gástrico ${ }^{38}, \mathrm{y}$ cáncer de cabeza y cuello ${ }^{39}$, se ha encontrado una correlación positiva entre un aumento de densidad del infiltrado inflamatorio y la progresión neoplásica.

Se ha reportado que la respuesta inflamatoria juega un papel importante en la regulación del desarrollo y crecimiento de lesiones precancerosas orales, debido a que modula el equilibrio entre el potencial inherente de crecimiento de la lesión precancerosa y su inhibición ${ }^{40}$.
Las limitaciones de nuestro estudio incluyen el carácter retrospectivo del análisis y el número limitado de casos, lo que posiblemente influyó para que el bajo escore del patrón de invasión no alcanzara valores estadísticamente significativos. No obstante, nuestros resultados sugieren que la ubicación de la lesión es un factor importante en el pronóstico de la enfermedad y que una respuesta inmune/inflamatoria adecuada, expresada en un bajo escore de RI, mejora el pronóstico de SV en pacientes con CCECO.

\section{Referencias}

1. Mehrotra R, Yadav S. Oral Squamous cell carcinoma: ethiology, pathogenesis and prognostic value of genomic alterations. Indian J Cancer 2006; 43: 60-66.

2. Walker DM, Boey G, McDonald LA. The pathology of oral cancer. Pathology 2003; 35: 376-383.

3. Leite IC, Koifman S. Survival analysis in a sample of oral cancer patients at a reference hospital in Rio de Janeiro, Brazil. Oral Oncol 1998; 34: 347-352.

4. Sargeran K, Murtomaa H, Safavi SM, Vehkalahti MM, Teronen O. Survival after diagnosis of cancer of the oral cavity. Br J Oral Maxillofac Surg 2008; 46: 187-191.

5. Goldenberg D, Brooksby C, Hollenbeak CS. Age as a determinant of outcomes for patients with oral cancer. Oral Oncol 2009; 45: e57-61.

6. Bànkfalvi A, Piffkò J. Prognostic and predictive factors in oral cancer: the role of the invasive tumour front. J Oral Pathol Med 2000; 29: 291-298.

7. Kaminagakura E, Vartanian JG, da Silva SD, Dos Santos CR, Kowalski LP. Case control study on prognostic factors in oral squamous cell carcinoma in young patients. Head Neck 2010; [Epub ahead of print].

8. Oliveira LR, Ribeiro-Silva A, Costa JP, Simões AL, Matteo MA, Zucoloto S. Prognostic factors and survival analysis in a sample of oral squamous cell carcinoma patients. Oral Surg Oral Med Oral Pathol Oral Radiol Endod 2008; 106: 685-695.

9. Kurokawa H, Yamashita Y, Takeda S, Zhang M, Fukuyama H, Takahashi T. Risk factors for late cervical lymph node metastases in patients with stage I or II carcinoma of the tongue. Head Neck 2002; 24: 731-736.

10. Costa Ade L, Araújo Júnior RF, Ramos CC. Correlation 
between TNM classification and malignancy histological feature of oral squamous cell carcinoma. Braz J Otorhinolaryngol 2005; 71: 181-187.

11. Bryne M, Koppang HS, Lilleng R, Stene T, Bang G, Dabelsteen E. New malignancy grading is a better prognostic indicator than Broders' grading in oral squamous cell carcinomas. J Oral Pathol Med 1989; 18: 432-437.

12. Högmo A, Kuylenstierna R, Lindholm J, MunckWikland E. Predictive value of malignancy grading systems, DNA content, p53 and angiogenesis for stage I tongue carcinomas. J Clin Pathol 1999; 52: 35-40.

13. Kristensen GB, Abeler VM, Risberg B, Trop C, Bryne M. Tumor size, depth of invasion, and grading of the invasive tumor front are the main prognostic factors in early squamous cell cervical carcinoma. Gynecol Oncol 1999; 74: 245-251.

14. Coussens LM, Werb Z. Inflammation and cancer. Nature 2002; 420: 860-867.

15. Heller DS, Hameed M, Cracchiolo B, Wiederkehr M, Scott D, Skurnick J, et al. Presence and quantification of macrophages in squamous cell carcinoma of the cervix. Int J Gynecol Cancer 2003; 13: 67-70.

16. Douglas WG, Tracy E, Tan D, Yu J, Hicks WL Jr, Rigual NR, et al. Development of head and neck squamous cell carcinoma is associated with altered cytokine responsiveness. Mol Cancer Res 2004; 2: 585-593.

17. Coussens L, Werb Z. Inflammatory Cells and Cancer: Think Different! J Exp Med 2001; 193: F23-26.

18. Bryne M, Jenssen N, Boysen M. Histological grading in the deep invasive front of $\mathrm{T} 1$ and $\mathrm{T} 2$ glottic squamous cell carcinomas has high prognostic value. Virchows Arch 1995; 427: 277-281.

19. Bryne M, Nielsen K, Koppang HS, Dabelsteen E. Reproducibility of two malignancy grading systems with reportedly prognostic value for oral cancer patients. J Oral Pathol Med 1991; 20: 369-372.

20. Costa Ade L, Pereira JC, Nunes AA, Arruda Mde L. Correlation between TNM classification, histological grading and anatomical location in oral squamous cell carcinoma. Pesqui Odontol Bras 2002; 16: 216-220.

21. Kane SV, Gupta M, Kakade AC, D' Cruz A. Depth of invasion is the most significant histological predictor of subclinical cervical lymph node metastasis in early squamous carcinomas of the oral cavity. Eur J Surg Oncol 2006; 32: 795-803.

22. Tumuluri V, Thomas GA, Fraser IS. The relationship of proliferating cell density at the invasive tumour front with prognostic and risk factors in human oral squamous cell carcinoma. J Oral Pathol Med 2004; 33: 204-208.

23. Guimarães GC, Lopes A, Campos RS, Zequi Sde C, Leal ML, Carvalho AL, et al. Front pattern of invasion in squamous cell carcinoma of the penis: new prognostic factor for predicting risk of lymph node metastases. Urology 2006; 68: 148-153.

24. Choi KK, Kim MJ, Yun PY, Lee JH, Moon HS, Lee TR, et al. Independent prognostic factors of 861 cases of oral squamous cell carcinoma in Korean adults. Oral Oncol 2006; 42: 208-217.

25. Woolgar JA. Histopathological prognosticators in oral and oropharyngeal squamous cell carcinoma. Oral Oncol 2006; 42: 229-239.

26. Zini A, Czerninski R, Sgan-Cohen HD. Oral cancer over four decades: epidemiology, trends, histology, and survival by anatomical sites. J Oral Pathol Med 2009; [Epub ahead of print].

27. Sarbia M, Bittinger F, Porschen R, Dutkowski P, Willers R, Gabbert HE. Prognostic value of histopathologic parameters of esophageal squamous cell carcinoma. Cancer 1995; 76: 922-927.

28. Muñoz-Guerra MF, Capote Moreno AL, Gómez Marazuela EM, Gamallo Amat C. Estadios precoces de cáncer oral: pronóstico en relación con gradación histológica, linfagiogénesis intratumoral y expresión de factor de crecimiento endotelial vascular Tipo-C (VEGF-C). Rev Esp Cirug Oral y Maxilofac 2006; 28: 25-40.

29. Crissman JD, Liu WY, Gluckman JL, Cummings G. Prognostic value of histopathologic parameters in squamous cell carcinoma of the oropharynx. Cancer 1984; 54: 2995-3001.

30. Kademani D, Bell RB, Bagheri S, Holmgren E, Dierks E, Potter B, et al. Prognostic factors in intraoral squamous cell carcinoma: the influence of histological grade. J Oral Maxillofac Surg 2005; 63: 1599-1605.

31. López Pizarro VM. Factores pronósticos de las metástasis linfáticas cervicales del carcinoma epidermoide de cavidad oral. Análisis de regression logística. Rev Esp Cirug Oral y Maxilofac 2000; 24: 161-170.

32. Einenkel J, Braumann UD, Horn LC, Pannicke N, Kuska JP, Schütz A, et al. Evaluation of the invasion front pattern of squamous cell cervical carcinoma by measuring classical and discrete compactness. Comput Med Imaging Graph 2007; 31: 428-435.

33. Eder T, Mayer R, Langsenlehner U, Renner W, Krippl $\mathrm{P}$, Wascher TC, et al. Interleukin-10 [ATA] promoter haplotype and prostate cancer risk: a population-based study. Eur J Cancer 2007; 43: 472-475.

34. Vose BM, Moore M. Human tumor-infiltrating lymphocytes: a marker of host response. Semin Hematol 1985; 22: $27-40$.

35. Oliveira-Neto HH, Leite AF, Costa NL, Alencar RC, Lara VS, Silva TA, et al. Decrease in mast cells in oral squamous cell carcinoma: possible failure in the migration of these cells. Oral Oncol 2007; 43: 484-490.

36. Black MM, Barclay THC, Hankey BFH. Prognosis in breast cancer utilizing histologic characteristics of the primary tumor. Cancer 1975; 36: 2048-2055.

37. Van Nagel JR, Donaldson ES, Wood EG, Parker JC. The significance of vascular invasion and lymphocytic infiltration in invasive cervical cancer. Cancer 1978; 41: 228-234. 
O. ARRIAGADA C. y cols.

38. Black MM, Freeman C, Torbjorn M, Harvei S, Cutler S. Prognostic significance of microscopic structure of gastric carcinomas and their regional nodes. Cancer 1971; 27: 703-711.

39. Wolf GT, Hudson JL, Peterson KA, Miller HL, McClatchey KD. Lymphocyte subpopulations infiltrating squamous carcinomas of the head and neck: correlations with extent of tumor and prognosis. Otolaryngol Head Neck Surg 1986; 95: 142-152.

40. Radhakrishna Pillai M, Balaram P, Chidambaram S, Padmanabhan TK, Nair MK. Development of an immunological staging system to prognosticate disease course in malignant cervical neoplasia. Gynecol Oncol 1990; 37: 200-205. 